\title{
A Career of Unyielding Exploration: In Memory of lon C. Baianu (1947-2013)
}

\author{
Ronald Brown ${ }^{1}$ \& James F. Glazebrook ${ }^{2,3}$ \\ 1 School of Computer Science, Bangor University, Gwynedd, United Kingdom. E-mail: r.brown@bangor.ac.uk \\ ${ }^{2}$ Department of Mathematics and Computer Science, Eastern Illinois University, Charleston, Illinois, USA. \\ E-mail: jfglazebrook@eiu.edu \\ ${ }^{3}$ Department of Mathematics, University of Illinois at Urbana-Champaign, Urbana, Illinois, USA
}

Editors: Danko Georgiev \& Christina Baianu

Article history: Submitted on April 7, 2013; Accepted on April 20, 2013; Published on May 15, 2013.

W e were deeply saddened to learn of the sudden death of our colleague, friend, and member of the Editorial Board of Quanta, Professor Ion C. Baianu, who unexpectedly passed away in Urbana, Illinois, USA, on February 10, 2013. Ion left behind his wife, Kimiko, his son, Stephen, and daughters, Antonia and Christina. He also left behind the achievements of a profound and illustrious career in his chosen fields of biophysics, spectroscopy, food science, and bioengineering.

Quanta 2013; 2: 1-6.

Ion was born on August 18, 1947 in Targu Logresti, Romania. He graduated in 1968 from the School of Physics and School of Medicine of the University of Bucharest (Romania). Following his graduate studies in Bucharest, he proceeded to obtain a doctoral degree from the University of London (Queen Elizabeth College) in 1974, and thereafter, an appointment as research fellow at the Cavendish Laboratory in Cambridge. While at the Cavendish, Ion shared a working association with several eminent scientists of the 20th century including the Nobel Laureate, Sir Nevill Mott. Surely such associations pro-

(c) (1) This is an open access article distributed under the terms of the Creative Commons Attribution License CC-BY-3.0, which permits unrestricted use, distribution, and reproduction in any medium, provided the original author and source are credited. vided the necessary inspiration for Ion's devotion to theoretical physics and spectroscopy throughout his career. After some time working in industry in the UK, he took up in 1980 joint appointments in the two Departments of Food Science and Human Nutrition, and of Nuclear Plasma and Radiological Engineering, as well as in the Near Infrared Reflectance Microspectroscopy Facility of the University of Illinois at Urbana-Champaign. During his tenure at the University of Illinois, Ion produced over 400 research articles and was editor and contributing author to more than 50 books. He was known to have been an excellent teacher, and an inspiring advisor to a number of graduate students who were privileged to work with him. His interest in presentation of the area of his Department is shown by his role as Editor of [1,2]. In recent years, Ion also pursued a number of large-scale and complex-interactive bioengineering projects, and of course also had the inevitable pressures and responsibilities of maintaining laboratories, along with the ongoing quest for institutional funding. It was typical of Ion's spirit of wide-scale scientific exploration, that during the course of his career he actively pursued a genuine interest in the mathematical methods of quantum mechanics, and in particular, the study of quantum automata and quantum computing. The origins of his interest, we imagine, would have been due to a surge of radical ideas in the field of the complexity of biological systems in the 1960s, 


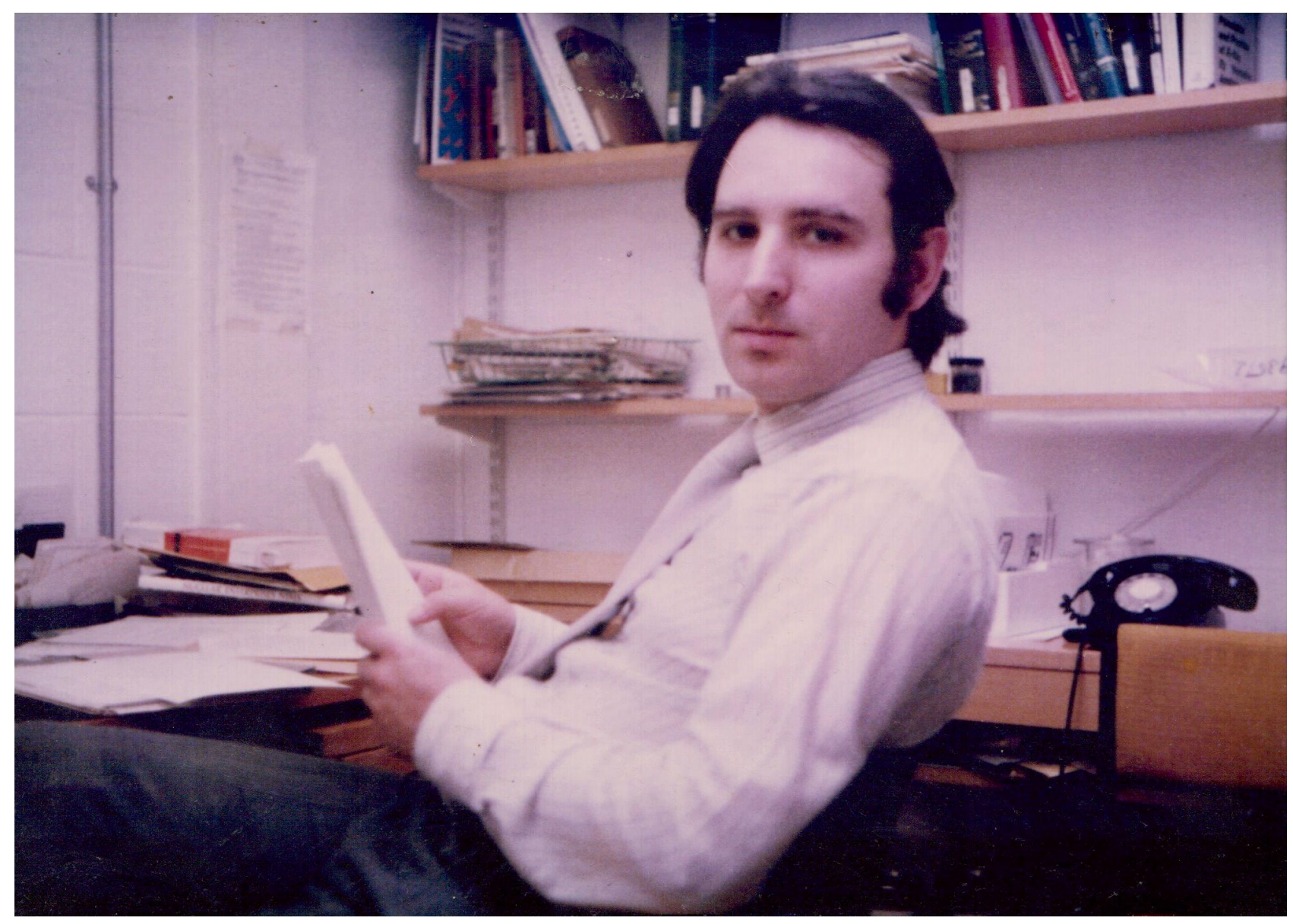

Figure 1: Ion Baianu in England, late 1970s.

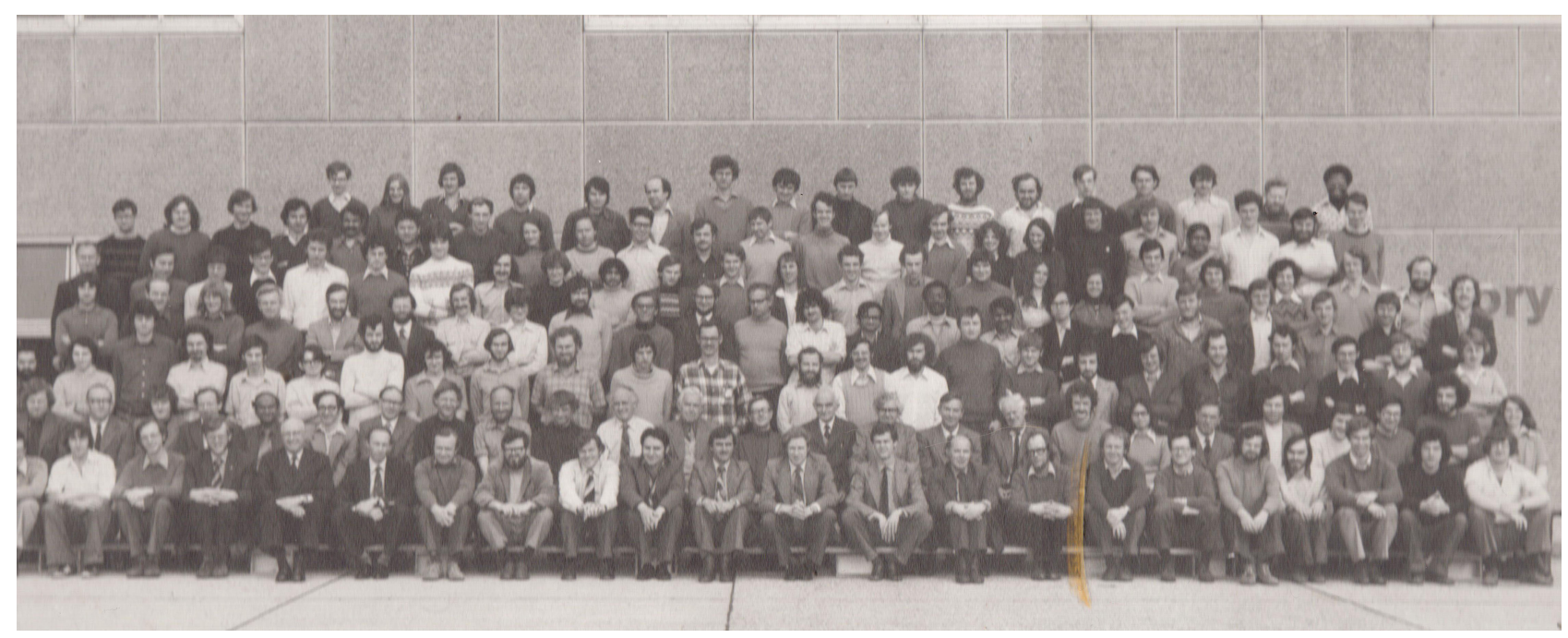

Figure 2: Cavendish Laboratory Physics Research Students in Cambridge, 1978. Ion Baianu is in the center of the first row.

and onwards. During this time, several of Ion's own contributions [3-7] (to name but a select few, including the earliest) were brought to the attention of a number of pioneering innovators in relational biology, such as Nicholas Rashevsky [8,9] and Robert Rosen [10-12] following the ground breaking work of Francis Crick and James
Watson, and others, who to some extent were inspired by Erwin Schrödinger's earlier thoughts on the fundamental nature of life [13]. The general aim was to establish a reliable categorical descriptive method that would embrace many of the natural complexity issues, and thus surpass the conventional reductionist practices of classical model- 


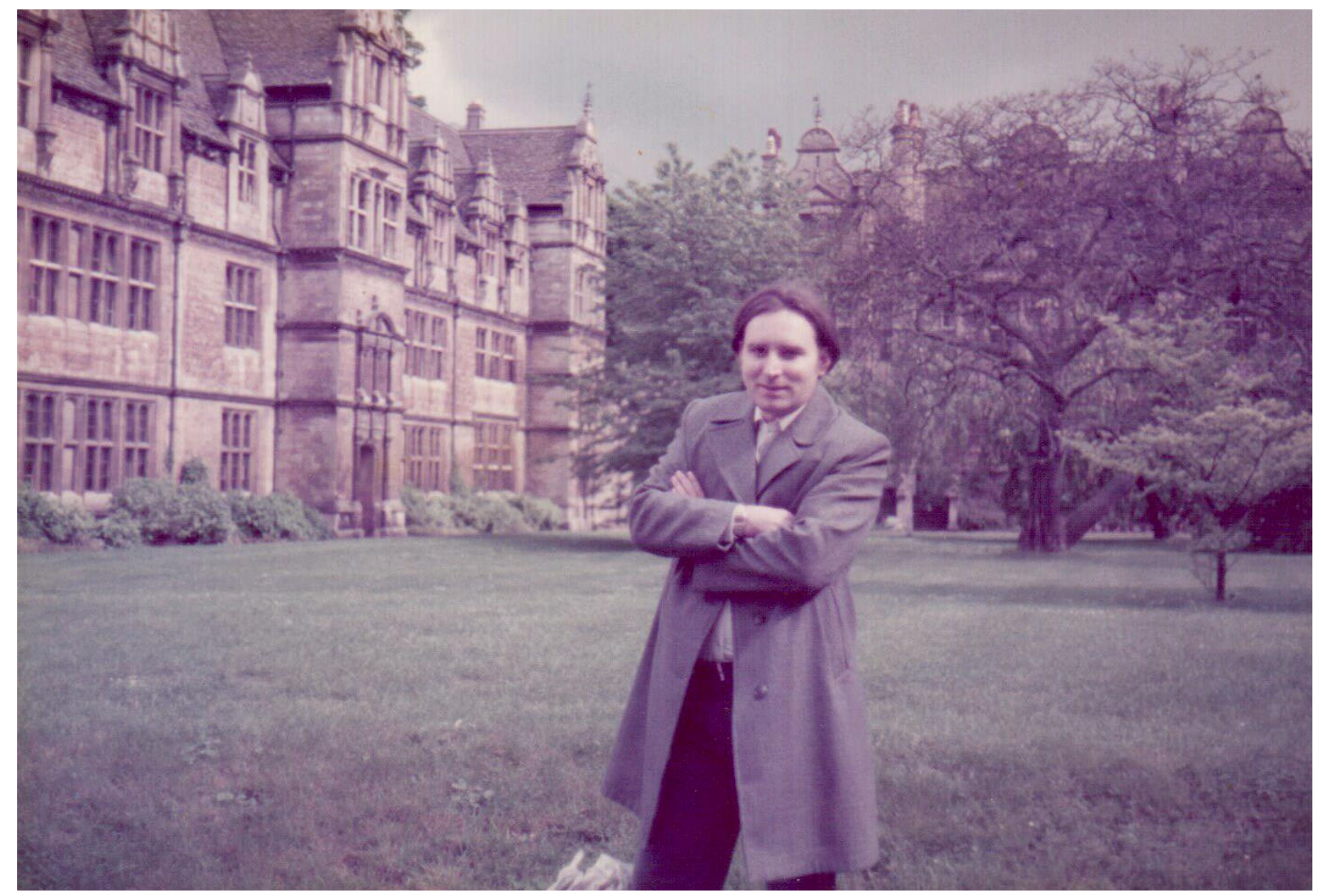

Figure 3: Ion Baianu in England, 1979.

ing and data analysis, that up to that time comprised much of the accepted methodology. The comparative value of Ion's contributions to this 'brave new world', as we came to be aware, was not just his encyclopedic knowledge of biological systems, but also in part due his knowledge of the abstract mathematical concepts of algebra, logic, topology, and category theory.

Ion had in Romania a rigorous secondary school and university instruction in mathematics, and maintained for much of his life a deep and passionate interest in the subject. As an example of his wide range of interests, we mention his translation from Romanian into English of the textbook Abelian Categories by the Romanian mathematician, Nicolai Popescu [14]. Perhaps from this work, he maintained an uncommon interest in the applications of categorical methods to applied areas, such as systems, and was fascinated by Robert Rosen's work on categories in biology. Also, among other achievements, Ion became a record-breaking contributor to the online resource, Planet Physics, by supplying a more than generous collection of entries on various concepts in mathematical physics, geometry and topology.

We had six joint publications with Ion, some of them lengthy, and of a survey type, and all showing his range of expertise. They came about in a curious way.
The first author (Ronald Brown) had pursued since 1965 some ideas of what came to be called, following his 1987 suggestion, "Higher Dimensional Algebra". One aspect was to find a mathematics which allowed 'algebraic inverse to subdivision', in the sense that in the following diagram:

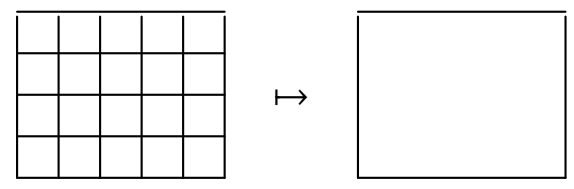

it seems reasonable to suggest that the right hand side should be some kind algebraic composition of the left hand side. Note that going from right to left in the diagram is subdivision, i.e. cutting up, standard technique in mathematics. But how do we express the result of putting things together again? This "gluing problem" is widespread in mathematics, physics, systems and biology. In our case, we need an analogue in dimension 2 of the usual algebraic composition $\left(a_{1}, a_{2}, \ldots, a_{n}\right) \mapsto a_{1} a_{2} \ldots a_{n}$ : that is, we need a transformation of a 2-dimensional array $\left(a_{i j}\right)$ into an actual "composition", say $\left[a_{i j}\right]$. This is allowed in the theory of double categories and groupoids, as Ronald Brown learned in 1965 from the book [15]. This was the start 


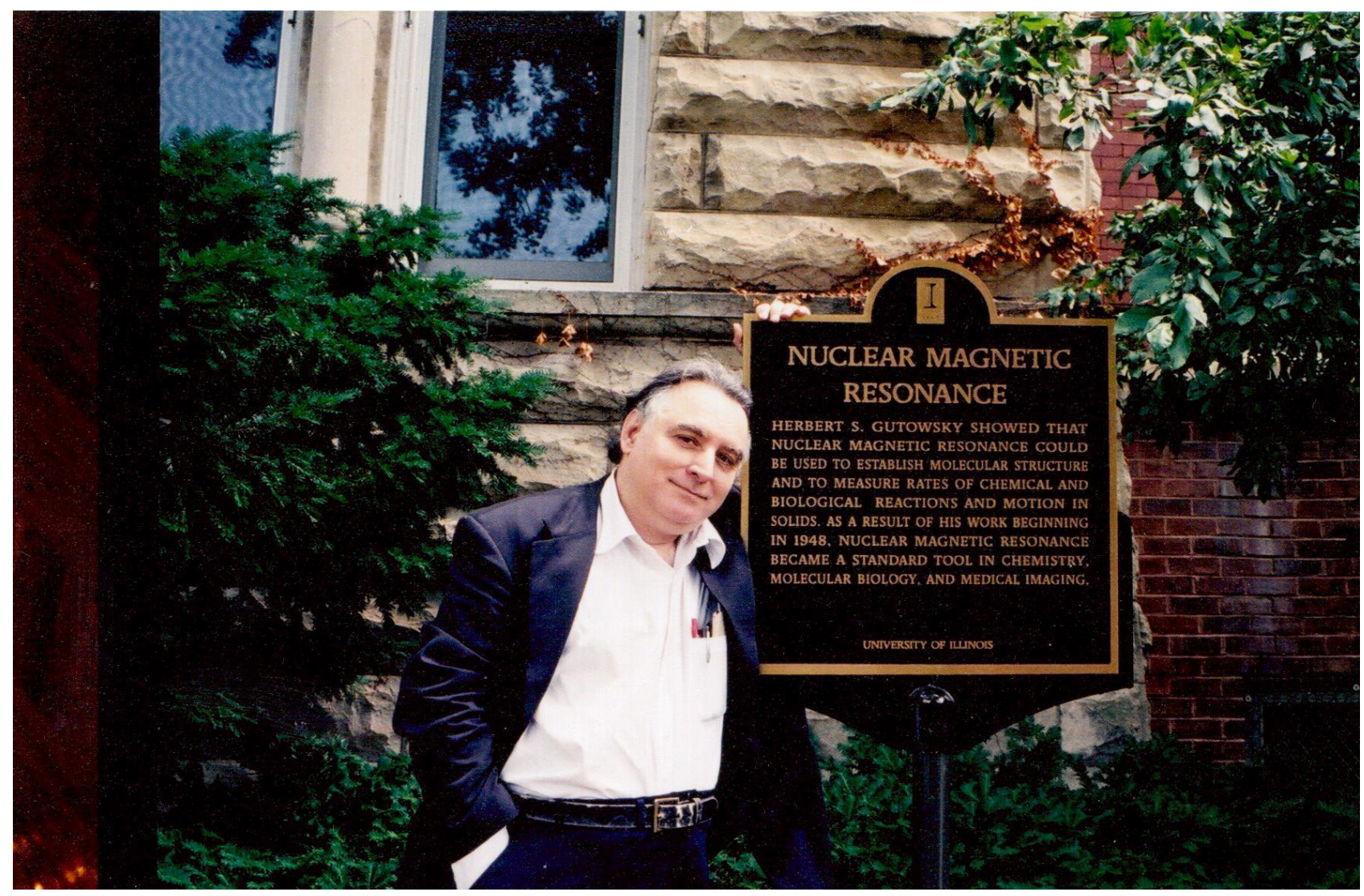

Figure 4: Ion Baianu in University of Illinios at Urbana-Champaign, 1997.

of the ideas of looking for the theory and applications of higher dimensional groupoids, considered in the spirit of group theory, namely leading to particular examples and to applications in algebraic topology. Indeed, considering the wide applications of group theory in mathematics and physics, it was natural to wonder about the potential applications of such a generalisation (the more mathematically inclined reader may wish to delve into the books [16, 17] for extensive details of the working concepts).

These ideas led to contact with workers in "concurrency theory" in computer science, where running several computers accessing databases can be considered as working in many time dimensions, and then to a seminar by Ronald Brown in G. Longo's series "Géometrie et Cognition" at the École Normale Supérieure in 2001, see [18]. This itself led to an invitation for Ronald Brown to give a talk to an International Conference on Theoretical Neuroscience at Delhi in 2003, which was published as [19]. The themes of this talk were: the categorical notion of colimit for expressing gluing properties, and for allowing distributed communication; and the idea that the brain certainly works in many dimensions, rather than just sequentially, i.e. on a line. Related ideas concerning such concepts as 'memory involutive systems' that employ category theory, can be seen in works such as [20,21], while [18 22 23] provide introductory surveys of the latter topic for non-specialists.

Ion made contact with the first author (Ronald Brown) in 2004 having found on Ronald Brown's web site the paper [19], which Ion felt linked with his longstanding work on categories in biological systems, and proposing a collaboration. Ronald Brown was then preparing to go to a meeting on "n-Categories: Foundations and Applications" at the Institute of Mathematics and its Applications (IMA) in Minnesota. However three years earlier the second author (James Glazebrook) had made contact with Ronald Brown to see if there could be a collaboration on groupoids in differential geometry, and this had led to an Science and Engineering Research Council (SERC) Visiting Fellowship for James Glazebrook to visit Bangor. Since Ion and James Glazebrook lived in Urbana (and James Glazebrook, coincidentally, being an adjunct faculty member at Ion's university), it was natural to suggest that James Glazebrook should be in contact with Ion, and it was a token of Ion's enthusiasm that the prospect of engaging in joint work soon came to the table. The key point was that the theory developed by Ronald Brown, as previously mentioned, could be applied to the structural patterning of evolving complex systems, and in this way one may see how the broader aspects of the approach 
enter into a philosophical/categorical framework. This led us to consider the work of several pioneering philosophers in this latter area, such as Franz Brentano, Edmund Husserl, Nicolai Hartmann, and others. In recent years, these philosophical investigations have been taken up by several proponents in the context of a Theory of levels, such as pursued by Ion's former collaborator, Roberto Poli (see e.g. [24] and references therein).

Another idea which fascinated Ion and is taken up in our joint papers as possibly related to evolution is the idea of iteration of local procedures, without requiring the notion of path to 'carry' these procedures, as happens for example in the usual process of analytic continuation. It is possible that this idea would lead to wider applications of non abelian groupoid like methods for local-to-global problems. For example, the following picture, taken from [25], illustrates a chain of local procedures from $a$ to $b$ :

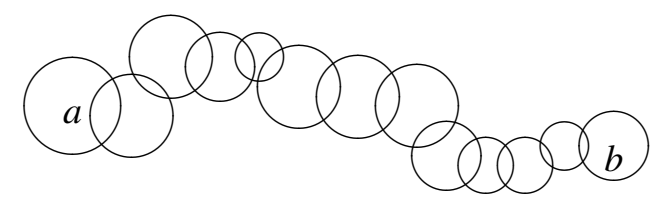

Needless to say, there is much that can be discussed concerning all of these topics, but for this obituary it is befitting now for us to simply list publications that resulted from our joint work [26-31].

We are grieved at the loss of Ion's acquaintance and friendship. He will always be remembered as a gifted, and profoundly energetic colleague with an unsatiable appetite for knowledge for which he was dedicated to impart on the basis of firm scientific value. We will all miss the extraordinary enthusiasm for life and science which he gave unstintingly to his family, his many colleagues, and his students.

\section{Acknowledgements}

The authors would like to thank Christina Baianu for checking the biographical information and for providing the photographs.

\section{References}

[1] Baianu IC. Physical Chemistry of Food Processes, Volume I: Fundamental Aspects. Springer, 1992.

[2] Baianu IC, Pressen H, Kumosinski TF. Physical Chemistry of Food Processes, Volume II: Advanced Techniques, Structures and Applications. Springer, 1993.
[3] Baianu IC, Marinescu M. Organismic supercategories: I. Proposals for a general unitary theory of systems. Bulletin of Mathematical Biophysics 1968; 30 (4): 625-635. http://cogprints.org/ $3831 /$

[4] Baianu IC. Some algebraic properties of (M, R)systems. Bulletin of Mathematical Biology 1973; 35 (1-2): 213-217. http://dx.doi.org/10.1007/ BF02558807

[5] Baianu IC. A logical model of genetic activities in Lukasiewicz algebras: The non-linear theory. Bulletin of Mathematical Biology 1977; 39 (2): 249-258. http://dx.doi.org/10.1007/ BF02462863

[6] Baianu I. Natural transformations of organismic structures. Bulletin of Mathematical Biology 1980; 42 (3): 431-446. http://dx.doi.org/10.1007/ BF02460794

[7] Baianu IC. Computer models and automata theory in biology and medicine. In: Mathematical Models in Medicine, vol.7, Witten M (editor), New York: Pergamon Press, 1987, pp.1513-1577. http: //cogprints.org/3687/

[8] Rashevsky N. Organismic sets and biological epimorphism. Bulletin of Mathematical Biophysics 1967; 29 (2): 389-393. http://dx.doi.org/10. $1007 /$ BF02476910

[9] Rashevsky N. Outline of a unified approach to physics, biology and sociology. Bulletin of Mathematical Biophysics 1969; 31 (1): 159-198. http: //dx.doi.org/10.1007/BFQ2478215

[10] Rosen R. A relational theory of biological systems. Bulletin of Mathematical Biophysics 1958; 20 (3): 245-260.http://dx.doi.org/10.1007/ BF02478302

[11] Rosen R. The representation of biological systems from the standpoint of the theory of categories. Bulletin of Mathematical Biophysics 1958; 20 (4): 317-341.http://dx.doi.org/10.1007/ BF02477890

[12] Rosen R. On complex systems. European Journal of Operational Research 1987; 30 (2): 129-134. http://dx.doi.org/10.1016/ 0377-2217(87)90089-0

[13] Schrödinger E. What is Life? Cambridge: Cambridge University Press, 1967. 
[14] Popescu N. Abelian Categories with Applications to Rings and Modules. New York and London: Academic Press, 1973. [English translation by Ion C. Baianu]

[15] Ehresmann C. Catégories et structures. Paris: Dunod, 1965.

[16] Brown R. Topology and Groupoids. Charleston, South Carolina: BookSurge Publishing, 2006.

[17] Brown R, Higgins PJ, Sivera R. Nonabelian Algebraic Topology: Filtered Spaces, Crossed Complexes, Cubical Homotopy Groupoids. European Mathematical Society Tracts in Mathematics, vol.15, European Mathematical Society, 2011.

[18] Brown R, Porter T. The intuitions of higher dimensional algebra for the study of structured space. Revue de Synthèse 2003; 124 (1): 173-203. http: //dx.doi.org/10.1007/BF02963404

[19] Brown R, Porter T. Category theory and higher dimensional algebra: potential descriptive tools in theoretical neuroscience. 2003: http://arxiv.org/ abs/math/0306223

[20] Ehresmann AC, Vanbremeersch JP. Hierarchical evolutive systems: A mathematical model for complex systems. Bulletin of Mathematical Biology 1987; 49 (1): 13-50. http://dx.doi.org/10. 1007/BFQ2459958

[21] Ehresmann AC, Vanbremeersch JP. The memory evolutive systems as a model of Rosen's organisms - (metabolic, replication) systems. Axiomathes 2006; 16 (1-2): 137-154. http://dx.doi.org/ $10.1007 / \mathrm{s} 10516-005-6001-0$

[22] Brown R, Paton R, Porter T. Categorical language and hierarchical models for cell systems. In: Computation in Cells and Tissues, Paton R, Bolouri H, Holcombe M, Parish JH, Tateson R (editors), Springer Berlin Heidelberg, 2004, pp.289-303. http://dx. doi.org/10.1007/978-3-662-06369-9_16

[23] Brown R, Porter T. Category Theory: an abstract setting for analogy and comparison. In: What Is Category Theory?, Sica G (editor), Monza, Italy: Polimetrica, 2006, pp.257-274.

[24] Baianu IC, Poli R. From simple to highly-complex systems: a paradigm shift towards non-abelian emergent system dynamics and meta-levels. Acta
Universitatis Apulensis 2012; (Special Issue on Understanding Complex Systems): 131-167. http://www.uab.ro/auajournal/acta2012/ No_10_Acta_Barni.pdf

[25] Brown R. Three themes in the work of Charles Ehresmann: Local-to-global; Groupoids; Higher dimensions. 2006. http://arxiv.org/abs/math/ 0602499

[26] Baianu IC, Brown R, Georgescu G, Glazebrook JF. Complex nonlinear biodynamics in categories: higher dimensional algebra, and LukasiewiczMoisil Topos: transformations of neuronal, genetic and neoplastic networks. Axiomathes 2005; 16 (1): 65-122. http://dx.doi.org/10.1007/ s10516-005-3973-8

[27] Baianu I, Brown R, Glazebrook J. Categorical ontology of complex spacetime structures: The emergence of life and human consciousness. Axiomathes 2007; 17 (3): 223-352. http://dx.doi.org/10. 1007/s10516-007-9011-2

[28] Baianu IC, Brown R, Glazebrook JF. A non-abelian, categorical ontology of spacetimes and quantum gravity. Axiomathes 2007; 17 (3): 353-408. http: //dx.doi.org/10.1007/s10516-007-9012-1

[29] Baianu IC, Glazebrook JF, Brown R. Algebraic topology foundations of supersymmetry and symmetry breaking in quantum field theory and quantum gravity: A review. Symmetry, Integrability and Geometry: Methods and Applications 2009; 5: 051. http://dx.doi.org/10.3842/SIGMA, 2009.051

[30] Baianu IC, Glazebrook JF, Brown R. Quantum symmetries, operator algebra and quantum groupoid representations: paracrystalline systems, topological order, supersymmetry and global symmetry breaking. International Journal of Research and Reviews in Applied Sciences 2011; 9 (2): 163-206. http://www. arpapress.com/ Volumes/Vol9Issue2/IJRRAS_9_2_01.pdf

[31] Brown R, Glazebrook JF, Baianu IC. A conceptual construction of complexity levels theory in spacetime categorical ontology: non-abelian algebraic topology, many-valued logics and dynamic systems. Axiomathes 2007; 17 (3-4): 409-493. http: //dx.doi.org/10.1007/s10516-007-9010-3 